\title{
Poliorquidismo: Aportación de un caso y revisión de la literatura actual
}

\author{
Velásquez López JG, Flórez Silva FR, López Montoya LJ. \\ Hospital Pablo Tobón Uribe. Medellín-Colombia \\ Actas Urol Esp 2006: 30 (4): 415-419
}

\section{RESUMEN}

POLIORQUIDISMO: APORTACION DE UN CASO Y REVISIÓN DE LA LITERATURA ACTUAL.

El poliorquidismo es una anomalía congénita urológica muy poco frecuente, con menos de cien casos publicados en la literatura mundial. Debe ser tenida en cuenta en todo estudio de masa escrotal. En ocasiones se encuentra asociado a otras entidades urológicas, entre ellas a malignidad, de ahí la importancia de su conocimiento y diagnóstico. Este último se logra confirmar mediante estudios imagenológicos, siendo los más importantes: la ecografia doppler y la resonancia nuclear, que nos permiten un preciso diagnóstico y ayudan a orientar el manejo definitivo. La posibilidad de malignidad en estos testículos supernumerarios (6\%), hace que algunos autores consideren en el tratamiento la extracción quirúrgica como única opción, pero reportes recientes indican que pacientes asintomáticos y con estudios imagenológicos normales se pueden observar. Se realiza el reporte de un caso en un paciente de 14 años diagnosticado por clínica e imagenología, del cual reportamos su manejo. Se realiza además una revisión de la literatura actual.

Palabras clave: Poliorquidismo. Duplicación testicular. Testículo supernumerario. Testículo. Anormalidades urogenitales.

\section{ABSTRACT}

POLYORCHIDISM: A CASE REPORT AND REVIEW OF THE LITERATURE.

Polyorchidism is an uncommon congenital defect. In fact, less than one hundred cases have been reported in the medical literature worldwide. This problem should be considered in the differential diagnosis of all scrotal masses. It is associated with multiple other urologic entities including malignancy. It is for this reason that it is important to be familiar with potential diagnostic and therapeutic strategies. This diagnosis is usually documented by imaging, specifically doppler ultrasound (US) and magnetic resonance imaging (MRI). The risk of malignancy approaches 6\% in Polyorchidism and therefore several authors have advocated surgery as the most appropriate therapeutic approach. However, recent reports suggest that asymptomatic patients with normal imaging could be observed. We present a 14 year old boy with polyorchidism which was diagnosed clinically and confirmed with imaging. His treatment and a review of the literature are presented as well.

Keywords: Polyorchidism. Testicular duplication. Supernumerary testis. Testis. Urogenital abnormalities.

$\mathrm{E}^{1}$ 1 poliorquidismo (PQ) es definido como la presencia de más de dos testículos. Su localización puede ser intra o extra escrotal. Es una alteración urológica congénita poco frecuente, con menos de cien casos reportados en la literatura mundial. Ocurre principalmente en el lado izquierdo y esta asociado en cantidades varia- bles a otras anomalías urogenitales entre ellas a malignidad, de allí la importancia de su conocimiento y presentación. Se reporta el caso de un paciente de 14 años diagnosticado por clínica e imagenología, del cual reportamos su manejo. Se realiza además una revisión de la literatura actual. 


\section{REPORTE DEL CASO CLÍNICO}

Paciente masculino de 14 años, residente en la ciudad de Medellín, previamente asintomático quien es remitido al servicio de urología del Hospital Pablo Tobón Uribe con un diagnóstico de masa testicular izquierda. El paciente se palpó incidentalmente una masa testicular, indolora, en área escrotal izquierda, sin notar crecimiento de ésta con el tiempo, no relacionada con trauma local ni asociada a otros síntomas. Consultó inicialmente al médico de su localidad y le ordenaron un estudio ecográfico el cual demostró en el saco escrotal izquierdo dos imágenes redondeadas, bien definidas de tamaño, forma y ecogenicidad similar, con medidas en promedio $9 \times 11 \mathrm{~mm}$ (Fig. 1), que presentaban además un patrón doppler similar. Dicho estudio descartó lesiones en el epidídimo. Con este resultado sugieren descartar masa testicular o testículo supernumerario y lo remiten al médico internista, quien le ordena una resonancia nuclear magnética (RNM), la cual reportó presencia de tres estructuras bien definidas de aspecto ovalado en saco escrotal, compatibles con dos testículos y uno adicional (ver Fig. 2), sin masas ni adenopatías. Por estos hallazgos lo remiten a urología. Paciente con antecedentes personales negativos. Al examen físico se encontró al paciente en buenas condiciones generales, con signos vitales dentro de los parámetros normales para la edad y un adecuado desarrollo pondoestural; en el abdomen no presentaba masas palpables ni

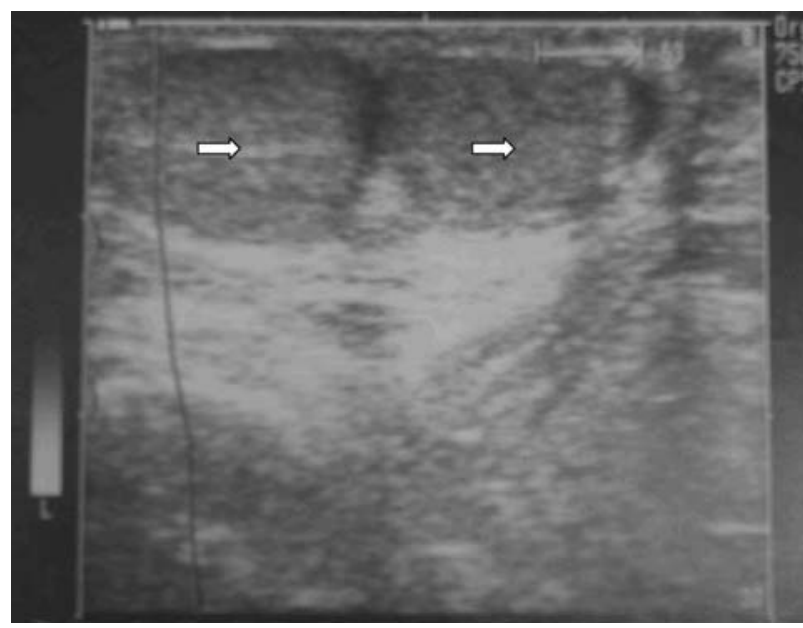

FIGURA 1. Imagen de la ecografia realizada inicialmente. Obsérvese las dos estructuras redondeadas presentes en el mismo hemiescroto (flechas).

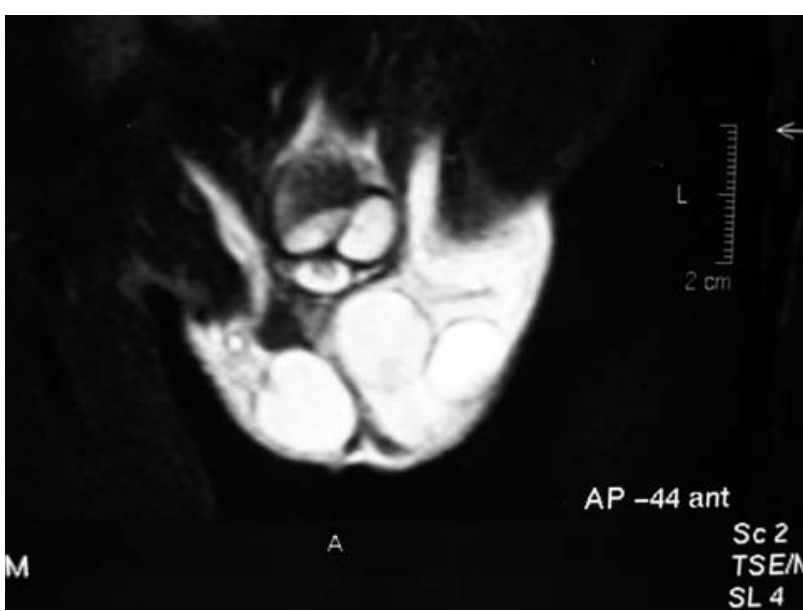

FIGURA 2. Imagen de la resonancia nuclear magnética del paciente, donde se observa la presencia de un triorquidismo.

hernias, y tampoco tenía adenopatías inguinales. En genitales externos, se observó el pene normal, y se palpan dos testículos sanos y además una masa esférica adenomatosa, localizada en área superior (proximal) del hemiescroto izquierdo, móvil no dolorosa, fácilmente separable con los dedos, sin presencia de nodulaciones ni signos de inflamación local.

Por la historia clínica, los estudios complementarios y los hallazgos al examen físico se hace una impresión diagnóstica de masa paratesticular o PQ. Con éste enfoque clínico y previa revisión del tema se decide llevar a cirugía para exploración y resección de dicha lesión, previo consentimiento informado del paciente y su familia. Bajo anestesia general se realiza una incisión escrotal transversa, con posterior disección por planos hasta la albugínea del lado izquierdo, observando la presencia de dos estructuras de igual tamaño, aspecto y consistencia compatibles con testículos, estando unidos por un epidídimo común y cada uno con un paquete vascular, donde solo uno, el de ubicación caudal, presentaba una estructura compatible con conducto deferente (Figs. 3 y 4), en este mismo se apreció un apéndice testicular, lo que nos llevó a pensar que correspondía en su origen embriológico al polo superior testicular, mientras que en el otro, de localización proximal o superior, se observó tenía un paquete venoso muy dilatado y único (Figs. 3 y 4). Se realizó sección, ligadura con hemostasia exhaustiva y corte de este último; se obtiene una estructura redondeada color carmelita, de 

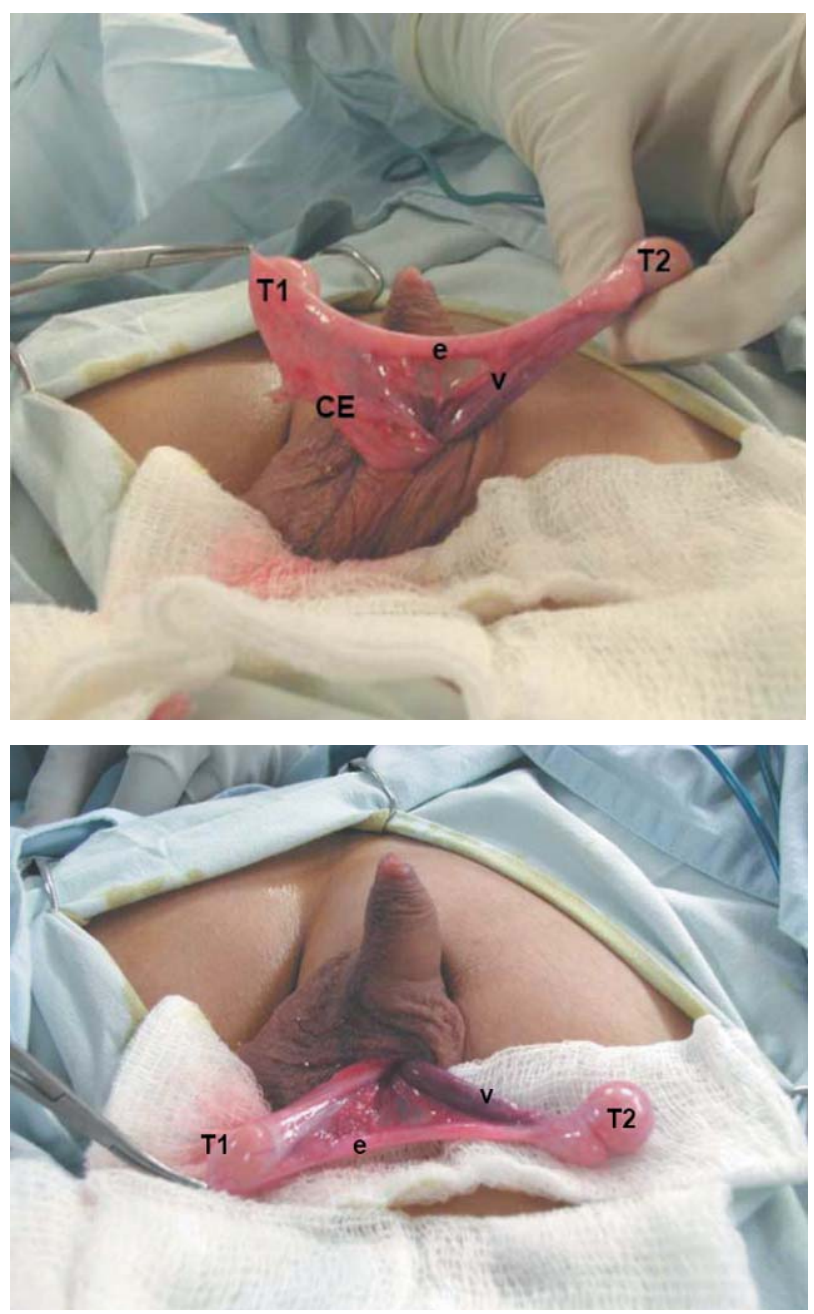

FIGURAS 3 y 4. Fotografias durante el acto operatorio. Se observan los dos testiculos del lado izquierdo (T1-T2) unidos por un epididimo común (e). Nótese la estructura venosa única (v) del testiculo supernumerario (T2), mientras que el testiculo de localización caudal (T1) presenta un cordón espermático con sus estructuras normales (CE).

consistencia firme, de aproximadamente $1.2 \mathrm{~cm}$ en su diámetro mayor, la cual se envía para estudio anatomopatológico. Luego se realiza bolsillo en espacio subdartos donde se aloja el testículo sano y es fijado con material de sutura orgánico absorbible (poliglactina) Vicryl ${ }^{\circledR}$ 4-0 (Ethicon, Johnson \& Johnson). Se realiza un cierre por planos y se procede a un bloqueo del nervio ilioinguinal del lado izquierdo. Se da de alta en su postoperatorio inmediato y evoluciona satisfactoriamente, sin presentar complicaciones. Para el informe de patología se procesan dos fragmentos y en los cortes histológicos se reporta un testículo normal. Sin neoplasia intratubular seminal.

\section{DISCUSIÓN}

Entre la cuarta y sexta semana del desarrollo embriológico y derivada del mesodermo, se inicia la formación de la cresta urogenital primitiva, que origina posteriormente el primordio testicu$\operatorname{lar}^{2}$. El epidídimo y conducto deferente provienen del conducto mesonéfrico de Wolf ${ }^{1,2}$. No se conocen los mecanismos exactos que explican la ocurrencia de los testículos supernumerarios pero parece existir una accidental elongación y fragmentación transversa o longitudinal de la cresta urogenital con o sin mesonefros antes de la octava semana de gestación. Dependiendo del plano de segmentación y del sitio de la misma se pueden desarrollar testículos supernumerarios con deferentes y epidídimos comunes o únicos ${ }^{3,4,21}$. Tampoco se ha encontrado una anomalía cromosómica para el $\mathrm{PQ}^{16,20}$.

El primer reporte de PQ fue descrito en 1670, sin embargo el primer caso comprobado histológicamente no se reportó hasta $1895^{9}$. Se presenta más comúnmente en la niñez o en la adolescencia y clínicamente puede manifestarse con dolor escrotal leve e intermitente asociado a edema local, y posteriormente puede presentar agudización e intensificación del mismo, con hallazgo de masa dura, de localización escrotal, inguinal o abdominal. Las complicaciones a las que puede estar asociado el PQ son: quistes, testículos retráctiles por reflejo cremastérico aumentado, criptorquidia $(40 \%)$, hernia $(30 \%)$, torsión (15\%), hidrocele $(9 \%)$, malignidad $(6 \%$ : teratoma, seminoma, rabdomiosarcoma o carcinoembrionario), fertilidad persistente luego de una vasectomía o infertilidad ${ }^{5-8,14,19}$. Estas complicaciones ocurren principalmente en el lado en el que ocurre la duplicación.

Lo más común es el hallazgo de un triorquidismo, generalmente en el lado izquierdo (>60\%) con evidencia de mayor tamaño y una posición craneal o proximal y anterior, con respecto al testículo normal ${ }^{10,14}$. Cuando se presenta con criptorquidia o malignidad es más común en el lado derecho ${ }^{9}$. Aunque existe el reporte de un caso con 5 testículos ${ }^{24}$, La presentación con duplicación bilateral es extremadamente rara y solo existen reportados menos de 10 casos $^{13}$. La presencia de un apéndice testicular, que son remanentes del conducto de Wolf, nos deben hacer pensar 
siempre que se trata del polo superior testicular por su desarrollo embriológico y por esto es el que tiene mayor probabilidad de tener conductos de salida, derivados de los túbulos mesonéfricos y por ende potencial espermatogénico ${ }^{20}$.

Existen dos clasificaciones ampliamente reconocidas en la literatura: una basada en el enfoque funcional y desarrollo embriológico, dónde el tipo I corresponde a un testículo supernumerario sin epidídimo ni deferente; el tipo II a un testículo supernumerario ligado al normal por un epidídimo común y comparte con él su deferente y en el tipo III el testículo supernumerario tiene su propio epidídimo y comparte el deferente con el testículo normal. La otra clasificación es la de Singer ${ }^{12}$, y es basada en la topografia, la anatomía y el potencial reproductivo del testículo; donde tipo I, es el testículo supernumerario que tiene conductos de salida para drenar al epidídimo y deferente, a su vez se subdivide en I A con posición intraescrotal y I B con posición ectópica (inguinal o abdominal); El tipo II, se corresponde con un testículo sin potencial reproductivo porque carece de los conductos de salida hacia el epidídimo y deferente; dividido así, el II A intraescrotal y el II B ectópico ${ }^{10-12}$.

Nuestro caso es un tipo II según la clasificación de Thum y I A según la clasificación de Singer. Cabe anotar que tanto el paciente como su madre deseaban la extirpación quirúrgica de la lesión y posterior estudio patológico.

Existen diagnósticos diferenciales del PQ, entre ellos están el espermatocele, hidrocele, quiste de epidídimo, epidídimo aberrante y quistes del cordón espermático (quistes de Nuck) que pueden simular una masa intraescrotal ${ }^{10}$.

Anteriormente el diagnóstico se realizaba por exploración quirúrgica y confirmación histológi$\mathrm{ca}^{6}$. Sólo con el examen físico no es segura la realización del diagnóstico exacto ${ }^{23}$. Actualmente se cuenta con estudios imagenológicos como la ecografia Doppler y RNM, que nos permiten un preciso diagnóstico e incluso ayudan a orientar el tipo de manejo definitivo. A pesar de no ser un método infalible, la ecografia doppler puede proporcionar ayuda importante al evaluar adecuadamente la presencia o la ausencia del flujo sanguíneo, si existe alteración en la homogeneidad ecogénica de los mismos y si hay presencia de masas tumorales ${ }^{22}$. La RNM tiene la ventaja de ser multiplanar, tener una alta resolución y contraste en los tejidos blandos y además de no ser operador dependiente aporta más detalles anatómicos, ideal para la adecuada localización de los testículos supernumerarios. También esta descrito la utilidad de la gamagrafia ${ }^{23}$ en el diagnóstico, la mayoría de los reportes la sugieren cuando existe sospecha de torsión de manera conjunta, su sensibilidad para evaluar la perfusión arterial con radiotrazador es buena pero se limita cuando el tamaño es pequeño o existe torsión crónica $^{25}$.

Debido a que uno de los principales puntos a evaluar en un testículo supernumerario es la presencia de malignidad, estos métodos diagnósticos se convierten en un arma fundamental a la hora de estudiar el paciente. En nuestro caso al paciente se le ordenó ecografía y RNM, que no reportaron presencia de masa y/o lesiones metastásicas sospechosas. Existen también otros métodos diagnósticos complementarios para evaluar su presencia o la probabilidad de malignidad en estos testículos, y son las pruebas hormonales: testosterona y hormona folículo-estimulante (FSH), marcadores tumorales: Alfa Fetoproteína y hormona gonadotropina coriónica subunidad B (BHCG), el espermograma y por último la biopsia, que nos ayuda a evaluar la espermatogénesis y la presencia de focos de displasia ${ }^{13-16}$.

Hasta 1980 se realizaba orquidectomía del testículo accesorio en todos los $\operatorname{casos}^{8}$. Los reportes recientes indican que si el paciente está asintomático y se demuestra normalidad del testículo supernumerario clínicamente y por imagenología, puede evitarse la extracción quirúrgica, esto con fines de conservación de la fertilidad futura. Está reportado que estos testículos en un grupo etario con potencial espermatogénico, esta se presenta de manera activa hasta en el 50\% de los $\operatorname{casos}^{19}$, aunque hay que tener presente que en muchos de estos casos los testículos carecen de un conducto deferente ${ }^{9}$.

Si el paciente está sintomático y el testículo accesorio es normal, la fijación para evitar torsión es suficiente, sin necesidad de resección.

La posibilidad de malignización siempre existe en los testículos supernumerarios, hecho que algunos autores consideran suficiente para reali- 
zar siempre su extracción. Aunque la presencia de atrofia y aplasia germinal es muy comúnmente encontrada en los estudios histológicos, hay poca evidencia para los cambios displásicos ${ }^{19}$.

En general se considera que los testículos ectópicos tipo I B, II B y II A, deben extraerse ${ }^{10}$. Los I A deben resecarse si hay cambios displásicos en la biopsia, espermatogénesis ausente, sospecha de malignidad en las imágenes, deseo del paciente de tener un solo testículo ó si el seguimiento periódico es imposible de realizar ${ }^{12,17-20}$. Algunos autores también sugieren extraer aquellos de localización intraabdominal ${ }^{10}$.

\section{CONCLUSIÓN}

Aunque su presentación es muy rara, el PQ es una anomalía a considerar durante el estudio de masas escrotales. La RNM y la ecografia proveen elementos útiles para clarificar la presencia de esta entidad. El tratamiento usual incluye la exploración quirúrgica y la fijación para evitar el riesgo de torsión. Al existir la probabilidad de malignidad consideramos mandatorio su extracción quirúrgica. Solo si hay dudas acerca de la naturaleza de la masa intra o extra escrotal o desea conocerse y preservar la función espermatogénica del testículo supernumerario, se debe realizar una biopsia complementaria, que ayude a definir la necesidad de extracción o por el contrario se puede preservar y luego continuar un seguimiento periódico. La opinión del paciente y sus acudientes es válida a la hora de tomar la decisión terapéutica.

\section{REFERENCIAS}

1. Park JM. Normal and anomalous development of the urogenital system. En: Walsh PC, Retik AB, Vaughan ED Jr, Wein AJ, Partin AW, Novick AC et al. Campbell's Urology, 8th ed. 2002; WB Saunders, Philadelphia; 1737-1764.

2. Tanagho EA. Embryology of the Genitourinary system. En: Tanagho EA, McAninch JW. Smith's General Urology, 16th ed. 2004; The McGraw-Hill Companies; 18-30.

3. Crawford DS, Bastable JR. Triorchidism with torsion. Br J Urol 1989;63(5):553-554.

4. Olumi AF, Richie LP. Urologic Surgery. En: Townsend CM, Beauchamp RD, Evers BM, Mattox KL. Sabiston textbook of surgery. 17th ed. 2004. Elseiver. Philadelphia, Pennsylvania. 2283-2318.

5. O`sullivan DC, Biyani CS, Heal MR. Polyorchidism : causation and management (letter). Postgrad Med J 1995;7:317318 .
6. Spranger R, Gunst M, Kuhn M. Polyorchidism: A strange anomaly with unsuspected properties. J Urology. 2002; 168(1) 195-198.

7. Witte M, Kim ED, Lipshultz LI. Torsion in a triorchid testis. J Urol 1998;159(5):1694.

8. Khetan N, Torkington J, Jamison MH. Polyorchidism presenting as retractile testes. BJU Int 1999;83:524-526.

9. Abbasoglu L, Salman FT, Gun F, Asicioglu C. Polyorchidism presenting with undescended testis. Eur J Pediatr Surg 2004; 14:355-357.

10. Yeniyol CÖ, Nergiz N, Tuna A. Abdominal polyorchidism: A case report and review of the literature. International Urology and nephrology 2004;36:407-408.

11. Thum G. Polyorchidism: case report and review of the literature. J Urol 1991;145:370-372.

12. Singer BR, Donaldson JG, Jackson DS. Polyorchidism: Functional classification and management strategy. Urology 1992;39:384-388.

13. Deveci S, Aygun C, Agildere AM, Özkardes H. Bilateral double testis: Evaluation by Magnetic Resonance Imaging. Int J Urology 2004;11(9):813-815.

14. Hwang S, Aronoff DR, Leonidas JC. Case 82: Polyorchidism with torsion. Radiology 2005;235:433-435.

15. Mallafre JM, Janeiro MR, Corominas S, Ictech S, Ovalle R, Carretero $P$. Supernumerary testis. Communication of a case and review of the literature Arch Esp Urol. 1989 Mar; 42(2): 166-168.

16. Berger AP, Steiner H, Hoeltl L, Bartsch G, Hobisch A. Ocurrence of Poliorchidism in a young man. Urology 2002; 60(5):911vi-vii.

17. Hancock RA, Hodgings TE. Polyorchidism. Urology 1984; 24:303-307.

18. Richie JP. Neoplasms of the testis. En: Walsh PC, Retik AB, Vaughan ED, Wein AJ. Campbell's Urology, 7th ed. 1998; WB Saunders, Philadelphia; 2411-2452.

19. Wolf B, Youngson GG. Polyorchidism. Pediatr Surg Int 1998; 13: 65-66.

20. Lawrentschuk N, MacGregor RJ. Polyorchidism: a case report and review of the literature. ANZ J Surg 2004;74:11301132 .

21. Danrad R, Ashker L, Smith W. Polyorchidism: imaging may denote reproductive potential of accessory testicle. Pediatr Radiol 2004;34:492-494.

22. Chung TJ, Yao JW. Sonographic features of polyorchidism. J Clin Ultrasound 2002;30:106-108.

23. Pantuck AJ, Barone JG. Polyorchidism in an 11-Year-Old Boy. Infect Urol 1998;11(1):27-29.

24. Day GH, Pike C. One man with five testes: Report of case. JAMA 1918;71:2055-2056.

25. Pentyala S, Lee J, Yalamanchili P, Vitkun S, Ali Khan S. Torsion testicular: Review. J Low Genital Tract Disease 2001;5(1):138-147.

Dr. JG Velásquez López

E-mail: juangvl@gmail.com

(Trabajo recibido el 24 de octubre 2005) 